\title{
Clinical speech impairment in Parkinson's disease, progressive supranuclear palsy, and multiple system atrophy
}

\author{
S. Sachin, G. Shukla, V. Goyal, S. Singh, Vijay Aggarwal' ${ }^{1}$, Gureshkumar ${ }^{2}$, M. Behari \\ Departments of Neurology, ${ }^{1}$ ENT and ${ }^{2}$ Biostatistics, All India Institute of Medical Sciences, Ansari Nagar, New Delhi - 110029 , India.
}

\begin{abstract}
Context: Speech abnormalities are common to the three Parkinsonian syndromes, namely Parkinson's disease (PD), progressive supranuclear palsy (PSP) and multiple system atrophy (MSA), the nature and severity of which is of clinical interest and diagnostic value. Aim: To evaluate the clinical pattern of speech impairment in patients with $P D, P S P$ and MSA and to identify significant differences on quantitative speech parameters when compared to controls. Design and Setting: Cross-sectional study conducted in a tertiary medical teaching institute. Materials and Methods: Twenty-two patients with PD, 18 patients with PSP and 20 patients with MSA and 10 age-matched healthy controls were recruited over a period of 1.5 years. The patients were clinically evaluated for the presence and characteristics of dysarthria. This was followed by quantitative assessment of three parameters: maximum phonation time (MPT), semantic fluency and reading speed. The outcome measures were compared between the patient groups and with controls. Results: Patients with PD had hypophonic monotonous speech with occasional rushes of speech while patients with MSA and PSP had mixed dysarthria with ataxic and spastic elements respectively. All quantitative parameters were affected when compared to controls $(P$ values $<0.001$, 0.012 and 0.008 respectively). Maximum phonation time was significantly less in PSP when compared to MSA and PD $(P=0.015)$. Reading speed also showed a similar trend which was not statistically significant. Semantic fluency was comparable in all three groups. Conclusion: Dysarthria in PD, PSP and MSA have many overlapping but certain distinctive features as well which could serve as a diagnostic clue. Patients with PSP had profound speech impairment probably indicative of the more severe frontostriatial pathology.
\end{abstract}

Key words: Dysarthria, Multiple system atrophy, Parkinson's disease, progressive supranuclear palsy, speech, voice

Speech impairment in Parkinsonian syndromes causes considerable impact on patients' social and emotional well-being. ${ }^{[1]}$ Speech disorder seen in Parkinson's disease (PD) is hypokinetic dysarthria characterized by abnormalities like inability to maintain loudness, monotonous and harsh voice, articulation errors and reduced fluency. ${ }^{[2-4]}$ Progressive supranuclear palsy (PSP) subjects can have a harsh and strained voice with frequent articulatory errors, stuttering, palilalia and variable intensity and rate of speech along with or even outweighing the monotonous speech of Parkinsonism. ${ }^{[5]}$ Speech in multiple system atrophy (MSA) is characterized by reduced loudness, variable rate and loudness, imprecise consonants, reduced stress, mono-pitch, voice strain and harshness in varying combinations. ${ }^{[6]}$ The predominant type of dysarthria corresponds well to the subtypes of MSA namely cerebellar (MSA-C) and Parkinsonian (MSA-P). ${ }^{[3]}$

Voice analysis in PD patients has revealed reduced maximum phonation time (MPT) and diadochokinetic rate, decreased intensity and harmonic-to-noise ratio and a narrow frequency range as well as limited loudness variability, high modal pitch and breathiness of voice..$^{[7-9]}$ The speed of reading a passage has been found to be variable in PD subjects which is attributed to differences in number of pauses, mean pause length, mean phrase length and mean syllable duration. ${ }^{[2.10]}$ The present study is the first study directly comparing the speech impairment in PD, PSP and MSA. Qualitative evaluation related to articulation, phonation and fluency as well as objective assessments like MPT, reading speed were done with respect to controls to ascertain the nature and severity of speech impairment in the three disorders. Semantic fluency, although a measure of word production and active memory, was also evaluated for its corroborative value to clinical speech assessment.

\section{Materials and Methods}

The present study included three groups of patients and one group of healthy controls whose native language was Hindi. Consecutive patients were recruited from the movement disorder clinic of the All India Institute 
of Medical Sciences, New Delhi, India over a period of two years from October 2004 to December 2006. Patients with a clinical diagnosis of PD according to the UKPD Brain Bank diagnostic criteria for Parkinson's disease, ${ }^{[11]}$ MSA as per consensus diagnostic criteria for MSA, ${ }^{[12]}$ and PSP as per NINDS-SPSP criteria ${ }^{[13]}$ comprised the first, second and third group of patients respectively. Multiple system atrophy patients were subclassified as MSA-C and MSA-P based on the accepted clinical criteria.

The following inclusion criteria were adhered to for selection of patients:

1. Age between 40-70 years

2. Hoehn and Yahr Stages 1-5 in case of PD subjects

The presence of any three of the following was considered as sufficient for exclusion of patients from the study:

1. Dementia (DSM IV) $)^{[14]}$

2. Depression/Psychiatric illness (DSM IV) ${ }^{[14]}$

3. All secondary causes of Parkinsonism

The control subjects were healthy age- and educationmatched individuals recruited from the employee population of the institute. Informed consent was taken from all patients and healthy controls. The study was approved by the institute ethics committee. History, physical examination and speech assessment were done by a neurologist. The patients were interviewed for age of onset, duration of disease, first symptom, current symptoms, most disabling symptom, response to L-dopa, current treatment and dosage of individual drugs, and for coexistent medical illness/treatment. All patients were off medications for at-least $12 \mathrm{~h}$ at the time of examination. The Unified Parkinson's Disease Rating Scale (UPDRS) subscores and total score were estimated for all the subjects in each of the three patient groups. ${ }^{[15]}$ The activities of daily living were determined using the Schwab and England scale. ${ }^{[16]}$ All PD patients were staged according to the Hoehn and Yahr staging system. ${ }^{[17]}$

We included 22 patients with PD (14 males and eight females), 18 patients with PSP (14 males and four females) and 20 patients with MSA (15 males and five females), along with 10 healthy controls (six males and four females) for the study. Among patients who had PD, eight (36.4\%) had mild disease (HYS 1-2), Eleven (50\%) had moderate disease (HYS 2.5-3) and three (13.6\%) had severe disease (HYS 4-5). Of the 20 patients with MSA, 11 patients had MSA-P had nine had MSA-C The baseline characteristics and salient differences in clinical features of these three groups are presented in Table 1. Apart from the patient groups we included 10 healthy controls with a mean age of $58.0 \pm 8.2$ years.

\section{Speech assessment}

Speech assessment was done by a neurologist (SS)

Table 1: Demographic and clinical characteristics of patients with Parkinson's disease, multiple system atrophy and progressive supranuclear palsy

\begin{tabular}{|c|c|c|c|c|c|}
\hline Parameter & & PD & MSA & PSP & $P$ value \\
\hline Age (yrs) & & $60.27 \pm 6.75$ & $56.8 \pm 8.13$ & $61.89 \pm 4.51$ & 0.063 \\
\hline $\operatorname{Sex}(M / F)$ & & $14 / 8$ & $15 / 5$ & $14 / 4$ & 0.367 \\
\hline Age of onset (yrs) & & $54.32 \pm 12.34$ & $54.45 \pm 8.82$ & $58.78 \pm 4.21$ & 0.256 \\
\hline \multicolumn{6}{|l|}{ Duration of } \\
\hline disease (yrs) & & $4.23 \pm 4.12$ & $2.45 \pm 1.95$ & $3.11 \pm 1.84$ & 0.146 \\
\hline Side affected first & Right/Left/B/L & 13/8/1(59.2) & 3/7/10(15) & 2/3/13(11.1) & $<0.001$ \\
\hline $\begin{array}{l}\text { Time interval between involvement } \\
\text { of two sides (months) }\end{array}$ & & $44.32 \pm 46.84$ & $15.8 \pm 19.65$ & $10.61 \pm 16.26$ & $<0.001$ \\
\hline \multirow[t]{11}{*}{ Current symptoms n (\%) } & Tremor & $20(90.9)$ & $11(55.0)$ & $4(22.2)$ & $<0.001$ \\
\hline & Rigidity & $22(100)$ & $19(95)$ & $18(100)$ & 0.362 \\
\hline & Change in gait & $16(72.7)$ & $18(90.0)$ & $18(100)$ & 0.036 \\
\hline & Loss of balance & $5(36.4)$ & $15(75.0 \%)$ & $18(100)$ & $<0.001$ \\
\hline & Bradykinesia & $20(90.9)$ & $20(100)$ & $17(94.4)$ & 0.399 \\
\hline & Dysarthria & $15(68.2)$ & $17(85)$ & $16(88.9)$ & 0.210 \\
\hline & Falls & $3(13.6)$ & $7(35.0)$ & $15(83.3)$ & $<0.001$ \\
\hline & Hallucinations & $3(13.6)$ & $2(10)$ & 0 & 0.284 \\
\hline & Dyskinesias & $6(27.3)$ & 0 & 0 & 0.003 \\
\hline & Dystonia & $2(9.1)$ & 0 & 0 & 0.332 \\
\hline & Autonomic & $3(13.6)$ & $20(100)$ & $1(5.5)$ & $<0.001$ \\
\hline $\begin{array}{l}\text { Initial subjective response to L-Dopa } \\
\text { Initial subjective }\end{array}$ & & $70.91 \pm 18.81$ & $8.67 \pm 15.98$ & $9.44 \pm 15.89$ & $<0.001$ \\
\hline response to L-Dopa (\%) & & $357.27 \pm 219.03$ & $148.42 \pm 162.93$ & $230.56 \pm 180.79$ & 0.004 \\
\hline Treatment duration (months) & & $30.86 \pm 42.54$ & $9.39 \pm 15.53$ & $18.11 \pm 22.93$ & 0.089 \\
\hline UPDRS 3 off* & & $39.32 \pm 11.65$ & $35.10 \pm 10.47$ & $35.28 \pm 13.67$ & 0.438 \\
\hline UPDRS Total & & $55.32 \pm 17.27$ & $52.55 \pm 15.73$ & $57.11 \pm 18.59$ & 0.712 \\
\hline MMSE\# & & $29.18 \pm 1.33$ & $29.05 \pm 1.17$ & $26.83 \pm 1.75$ & $<0.001$ \\
\hline S and $E$ ADL $\$$ Score & & $74.09 \pm 20.39$ & $55.5 \pm 21.64$ & $57.22 \pm 21.09$ & 0.010 \\
\hline
\end{tabular}

*Unified Parkinson's disease rating scale 3 (off-phase); "Mini mental state examination, \$Schwabe and England Activities of Daily Living 
and a speech pathologist (VA) in one sitting. These assessments were done while patient was asked to narrate his/her history of illness and also during the reading of a passage (of 275 words) in Hindi. The session lasted from 15-20 min. The following deviant dimensions were looked for in the speech sample studied:

Hypophonia: reduced volume of speech

Mono-pitch: the speech is delivered at a constant pitch without normal modulations

Spastic speech: characterized by strained -strangled quality with imprecise consonants

Ataxic speech: characterized by excess and equal stress laid on ordinarily unstressed words or syllables, excess loudness or bursts of loudness with irregular articulatory breakdown.

Rushes of speech: sudden usually short duration acceleration of the rate of speech.

These components were recorded as present when the typical characteristics mentioned above were observed without further characterization. ${ }^{[18,19]}$

Three quantitative assessment tasks were also made in each participant:

1. Maximum phonation time (MPT) ${ }^{[8,10]}$ defined as the maximum time for which the patient was able to sustain phonation. For this, patients were instructed to take a deep breath and then sustain phonation [a] for as long as possible. Three samples were obtained and the best (longest) response was taken.

2. Semantic fluency: The number of names of animals a person was able to spontaneously report in one minute.

3. Reading speed: Speed of reading was assessed by asking the patient to read aloud a standard paragraph in Hindi language. The number of words read by the subject in one minute was recorded as his/her reading speed.

\section{Statistical analysis}

The data was analyzed by using SPPS (Chicago) Version 15.0. The significant differences in the demographic and clinical characteristics of patients pertaining to the three groups (PD, MSA and PSP) were determined using Chi-square and Fisher exact tests for categorical and continuous variables respectively. The speech parameters were assessed by parametric (oneway ANOVA) and non-parametric (Kruskal-Wallis) tests wherever applicable depending on the nature of the data (normal/not respectively), followed by multiple comparison tests (Bonferroni method). The controls were also included in the analysis for quantitative speech parameters. A $p$ value of less than 0.05 was considered to be significant.

\section{Results}

All the patients included in the present study in each of the three groups were found to have speech abnormalities on examination, but only 15 PD patients (68.2\%), 17 MSA (85\%) and 16 PSP patients (88.9\%) complained of some form of difficulty in speech or alteration of voice. Dysarthria was reported as the most disabling among all the symptoms in only one patient with PSP and two patients with PD. It was never the initial manifestation of illness in any of the patients. Patients with PSP reported dysarthria more often than those with MSA or PD, probably because of the early and more severe involvement of speech in these patients.

\section{Qualitative assessment}

The proportion of patients with hypophonia was significantly lower in the MSA group (80\%) compared to PD and PSP (100\% in both groups) $(p=0.014)$. A similar trend was seen for mono-pitch $(p=0.051)$. Rushes of speech were more common in the PSP group (39.9\%) but was also seen in the PD (22.7\%) and MSA (20\%) groups as well and the difference was not statistically significant. Ataxic speech was exclusively noticed in patients with MSA-C (100\%). Spastic speech was observed in a higher proportion of PSP patients (66.7\%) than PD (1\%) and MSA (15\%) $(\mathrm{p}<0.001)$. The results are summarized in Table 2.

\section{Objective assessment}

The patient groups when compared to controls differed significantly in the three speech parameters, namely MPT $(p<0.001)$, semantic fluency $(p=0.012)$ and the reading speed $(p=0.008)$ [Table 2]. On post-hoc analysis, PD and PSP subjects differed significantly from the controls in the MPT ( $p=0.008$ and $<0.001$ respectively).

\begin{tabular}{|c|c|c|c|c|c|}
\hline & $P D(n=22)$ & MSA $(n=20)$ & PSP (n=18) & Controls $(n=18)$ & $P$ value \\
\hline Hypophonia, n(\%) & $22(100)$ & $16(80)$ & 18(100) & & 0.014 \\
\hline Mono-pitch n(\%) & $20(90.9)$ & $15(75)$ & $18(100)$ & & 0.051 \\
\hline Rushes of speech $\mathrm{n}(\%)$ & $5(22.7)$ & $4(20.0)$ & $7(39.9)$ & & 0.361 \\
\hline Ataxic comp $\mathrm{n}(\%)$ & 0 & $10(50)$ & 0 & & $<0.001$ \\
\hline Spastic comp $n(\%)$ & $1(4.5)$ & $3(15)$ & 12(66.7) & & $<0.001$ \\
\hline Mean phonatory duration (mean \pm SD) & $9.59 \pm 3.55^{*}$ & $10.45 \pm 3.17$ & $7.33 \pm 2.99^{*}$ & $13.66 \pm 2.18$ & $<0.001$ \\
\hline Semantic fluency (mean $\pm S D$ ) & $12.45 \pm 3.64$ & $11.5 \pm 3.27$ & $10.44 \pm 3.69^{*}$ & $15.22 \pm 4.46$ & 0.012 \\
\hline Reading speed (mean $\pm S D)$ & $109.59 \pm 43.36$ & $114.55 \pm 42.76$ & $85.29 \pm 28.28 *$ & $147.33 \pm 17.94$ & 0.008 \\
\hline
\end{tabular}

*Significant difference with respect to controls on post-hoc tests; Figures in parentheses are in percentage 
For semantic fluency and reading speed the difference was significant only for the PSP group with respect to controls ( $p$ values $=0.009$ and 0.006 respectively).

The analysis was repeated in the patient groups (PD, MSA and PSP) after excluding the control group to examine for important differences between them. Significant overall differences were seen only for MPT $(P=0.015)$. On post-hoc tests, the difference in MPT was significant between the PSP and MSA groups $(p=0.014)$. The reading speed was affected the most in PSP, but the difference between groups was not significant. The semantic fluencies were comparable.

\section{Discussion}

In the present study we found speech impairment in all patients with PD, MSA and PSP on clinical examination. Parkinson's disease patients were noted to have hypophonic and monotonous speech while patients with MSA generally had mixed dysarthria with hypophonic component predominating in MSA-P and ataxic component in MSA-C; whereas in PSP, the speech abnormality was more severe and also had a mixed pattern of hypophonic and spastic components.

The pathophysiological basis of speech impairment is akin to the motor phenomena seen in PD. Laryngeal muscles in akinetic rigid syndromes become rigid and resistant to movement resulting in decreased vocal cord abduction, altered glottic pressures and poor coordination with respiration. This results in impaired phonation, inability to adequately intonate speech leading to decreased loudness, monotony and hoarseness of speech. ${ }^{[2,18]}$ Electromyographic studies have revealed abnormal firing of motor units occurring during rest in the thyroarytenoid and cricothyroid muscles commensurate with increased tone in these muscles. ${ }^{[20,21]}$ Central mechanism is suggested by a Positron Emission Tomogrophy (PET) study which showed increased activation during sustained phonation and a subsequent reduction in activity after voice therapy. ${ }^{[22]}$ Short rushes of speech seen in some patients is considered to be the same phenomenon as festination of gait seen in akinetic rigid syndromes. A case-control study which examined oral festination in $\mathrm{PD}$ found this phenomenon in $45 \%$ of subjects. ${ }^{[23]}$

Speech assessment in PSP patients revealed hypophonia and monotonous speech in all patients, short rushes of speech in $38.9 \%$ and spastic components in $66.7 \%$. In a previous study using quantitative perceptual speech analysis along with oral motor examinations, $50 \%$ of PSP patients had predominantly spastic, $34 \%$ hypokinetic and $14 \%$ ataxic components, and the rest with spastic, hypokinetic, and ataxic components were equal. ${ }^{[5]}$ Jellinger examining a similar group of PSP patients, reported mixed hypokinetic and spastic speech in $94 \%$, ataxic speech in $5 \%$ and pure hypokinetic speech in $1 \% .{ }^{[24]}$

The ataxic component was lacking in our patients with PSP. The cerebellar pathology in PSP is less severe compared to cortical and extrapyramidal involvement accounting for relative absence of ataxic component. ${ }^{[25]}$ The spastic nature of speech is due to bilateral cortico-bulbar involvement. Speech abnormalities in MSA patients included hypophonia in $80 \%$, monotonous speech in $75 \%$, and short rushes of speech in $20 \%$, ataxic speech in $50 \%$ of patients and spastic components in $15 \%$. The results were similar to a previous study. ${ }^{[6]}$

Objective assessments showed MPT to be significantly reduced in PSP followed by PD subjects. Comparable findings were seen in previous studies on PD subjects when compared to controls..$^{[2,7,8]}$ This aspect has not been studied earlier in PSP or MSA. The reduction in MPT may be a combined effect of reduced phonatory ability and poor respiratory effort secondary to limited synchronous movements of pectoral and diaphragmatic muscles. ${ }^{[2,3]}$ Semantic fluency was reduced in all patient groups compared to controls. Patients with PSP were maximally affected while those with PD performed better in this task. Several studies have reported category naming defects in $\mathrm{PD} .{ }^{[26-28]} \mathrm{A}$ metaanalysis has shown significantly more impairment of semantic fluency relative to phonemic fluency in patients with PD. ${ }^{[26]}$ Verbal fluency reduction has been reported in patients with PSP and MSA as well. ${ }^{[29-31]}$

Reduced reading speed was seen in all three patient groups when compared to controls, commensurate with bradykinesia. A previous study comparing reading speed in PD subjects with controls did not find any significant difference between the groups. ${ }^{[2]}$ Reduced reading speed could be due to inappropriate and or long pauses occurring during the course of reading, reduced phrase length and prolonged mean syllable duration. ${ }^{[11]}$ Microelectrode recoding of subthalamic nucleus during repetitive sentence repetition task has shown basal ganglia to have a significant role in meaningful speech utterances. ${ }^{[32]}$ The profound speech dysfunction seen with PSP may be due to greater loss of neurons in the substantia nigra ${ }^{[24,33]}$ and more severe frontal lobe dysfunction in these patients compared to MSA and PD. ${ }^{[29]}$

This is the first study comparing clinical disorders of speech in PD, PSP and MSA. Although there is no specific pattern of dysarthria for each of these disorders, the presence of some findings may give a clue to the diagnosis. Future studies should aim to explore the articulatory errors specific to these disorders in a larger study population with audiological and functional imaging correlation. 


\section{References}

1. Miller N, Noble E, Jones D, Burn D. Life with communication changes in Parkinson's disease. Age Ageing 2006;35:235-9.

2. Canter GJ. Speech characteristics of patients with Parkinson's disease: I, Intensity, Pitch, and Duration. J Speech Hear Disord 1963;28:221-9.

3. Canter GJ. Speech characteristies of patients with Parkinson's disease: II, Physiological support for speech. J Speech Hear Disord 1965;30:21724.

4. Critchley EM. Speech disorders of Parkinsonism: A review. J Neurol Neurosurg Psychiatry 1981;66:751-8

5. Kluin KJ, Gilman S, Lohman M, Junck L. Characteristies of the dysarthria of multiple system atrophy. Arch Neurol 1996;53:545-8.

6. Kluin KJ, Foster NL, Berent S, Gilman S. Perceptual analysis of speech disorders in progressive supranuclear palsy. Neurology 1993;43:563-6

7. Midi I, Dogan M, Koseoglu M, Can G, Sehitoglu MA, Gunal DI. Voice abnormalities and their relation with motor dysfunction in Parkinson's disease. Acta Neurol Scand 2008;117:26-34

8. Yücetürk AV, Yilmaz H, Eğrilmez M, Karaca S. Voice analysis and videolaryngostroboscopy in patients with Parkinson's disease. Eur Arch Otorhinolaryngol 2002;259:290-3.

9. Holmes RJ, Oates JM, Phyland DJ, Hughes AJ. Voice characteristics in the progression of Parkinson's disease. Int J Lang Commun Disord 2000;35:407-18.

10. Hammen VL, Yorkston KM. Hammen VL, Yorkston KM Speech and pause characteristics following speech rate reduction in hypokinetic dysarthria. J Commun Disord 1996;29:429-44.

11. Ward CD, Gibb WR. Research diagnostic criteria for Parkinson's disease. Adv Neurol 1990;53:245-9.

12. Gilman S, Low PA, Quinn N, Albanese A, Ben-Shlomo Y, Fowler CJ, \& al. Consensus statement on the diagnosis of multiple system atrophy. $J$ Neurol Sci 1999;163:94-8.

13. Litvan I, Agid Y, Calne D, Campbell G, Dubois B, Duvoisin RC, \& al. Clinical research criteria for the diagnosis of progressive supranuclea palsy (Steele-Richardson-Olszewski syndrome): Report of the NINDSSPSP international workshop. Neurology 1996;47:1-9.

14. In: Diagnostic and statistical manual of mental disorders, 4th ed. Primary care version. Washington, DC: APA; 1995.

15. Fahn S, Elton R. Unified Parkinson's disease rating scale. In: Fahn S, Marsden CD, Calne DB, Goldstein M, editors. Recent developments in Parkinson's disease. Vol. 2. Florham Park, N.J.: Macmillan Healthcare Information; 1987. p. 153-64.

16. Schwabe R, England A. Projection technique for evaluating surgery in Parkinsons disease. 3rd Symposium of Parkinson's disease. In: Gillingham F, Donaldson I, editors. Edinburgh Livingstone; 1969. p. 152-7.

17. Hoehn MM, Yahr MD. Parkinsonism: Onset, progression and mortality. Neurology 1967;17:427-42.

18. Corlton RH, Casper JK, Leonard R. Understanding voice problems: A physiological perspective for diagnosis and treatment. Lippincot Williams and Wilkins; 2006.

19. Darley FL, Aronson AE, Brown JR. Motor speech disorders. Philadelphia, Pa: WB Saunders Co; 1975.

20. Baker KK, Ramig LO, Luschei ES, Smith ME. Thyroarytenoid muscle activity associated with hypophonia in Parkinson disease and aging. Neurology 1998;51:1592-8.

21. Zarzur AP, Duprat AC, Shinzato G, Eckley CA. Laryngeal electromyography in adults with Parkinson's disease and voice complaints. Laryngoscope 2007;117:831-4.

22. Liotti M, Ramig LO, Vogel D, New P, Cook CJ, Ingham RJ, \& al Hypophonia in Parkinsons disease: Neural correlates of voice treatment revealed by PET. Neurology 2003;60:432-40.

23. Moreau C, Ozsancak C, Blatt JL, Derambure P, Destee A, Defebvre L. Oral festination in Parkinson's disease: Biomechanical analysis and correlation with festination and freezing of gait. Mov Disord 2007;22:1503-6

24. Jellinger K. Neuropathologic correlates of dysarthria in progressive supranuclear palsy. Arch Neurol 2001;58:1499-500.

25. Daniel SE, de Bruin VM, Lees AJ. The clinical and pathological spectrum of Steele-Richardson-Olszewski syndrome (progressive supranuclear palsy): A reappraisal. Brain 1995;118:759-70.

26. Henry JD, Crawford JR. Verbal fluency deficits in Parkinson's disease: A meta-analysis. J Int Neuropsychol Soc 2004;10:608-22.

27. Bayles KA, Trosset MW, Tomoeda CK, Montgomery EB Jr, Wilson J. Generative naming in Parkinson disease patients. J Clin Exp Neuropsychol 1993;15:547-62.

28. Azuma T, Bayles KA, Cruz RF, Tomoeda CK, Wood JA, MeGeagh A, đal. Comparing the difficulty of letter, semantic, and name fluency tasks for normal elderly and patients with Parkinson's disease. Neuropsychology 1997;11:488-97

29. Bürk K, Daum I, Rüb U. Cognitive function in multiple system atrophy of the cerebellar type. Mov Disord 2006;21:772-6.

30. Millar D, Griffiths P, Zermansky AJ, Burn DJ. Characterizing behavioral and cognitive dysexecutive changes in progressive supranuclear palsy. Mov Disord 2006;21:199-207.

31. Robbins TW, James M, Owen AM, Lange KW, Lees AJ, Leigh PN, et al. Cognitive deficits in progressive supranuclear palsy, Parkinson's disease and multiple system atrophy in tests sensitive to frontal lobe dysfunction. J Neurol Neurosurg Psychiatry 1994;57:79-88.

32. Watson P, Montgomery EB Jr. The relationship of neuronal activity within the sensori-motor region of the subthalamic nucleus to speech. Brain Lang 2006;97:233-40.

33. Kluin K, Gilman S, Foster N, Sima A, D'Amato C, Bruch L, \& al. Neuropathological correlates of dysarthria in progressive supranuclear palsy. Arch Neurol 2001;58:265-9.

Accepted on 15-04-2008

Source of Support: Nil, Conflict of Interest: None declared. 